\title{
Research on an Automatic Adjustment Control System for Small Platform Based on the PLC
}

\author{
Honjun Yang ${ }^{1}$ and Pan Zhu \\ ${ }^{1}$ School of Mechanical Engineering of Wuhan Polytechnic University, Changqin Guard Street, Wuhan, \\ China.
}

Key words: PLC, Small Platform, Level adjustment, Two-axis position adjustment

\begin{abstract}
Many engineering devices need a platform with high level and position accuracy. An automatic control system is proposed to adjust a small platform that is supported by four height-adjustable legs and two length-adjustable level screws. The control system scheme and working principle are analyzed, then the structure of the control system based on PLC is put forward and the hardware is designed. And then the direction decoupling control strategy is put forward and software of the control system is designed in detail. The device can adjust the levelness and position of the platform quickly, with the man-machine interface of electronic touch screen for status display and parameter setting, the functions of virtual leg detection and limit protection.
\end{abstract}

\section{INTRODUCTION}

Many devices need a level platform, for example vehicle-borne radar, static pile pressing machine and artillery launcher. For these devices, the level error of the platform must be adjusted before them are utilized normally [1]. In recent years, there have been many kinds of leveling methods for this platform including three points supported leveling, four points supported leveling and six points supported leveling. These leveling methods can be divided into two categories, namely, manual adjustment and automatic adjustment. According to the actuator, the automatic adjustment methods have two types, including the mechatronics and hydraulics [2]. At present, all kinds of leveling devices are mainly designed for large and medium-sized equipment. On the other hand, for some small equipment, such as measuring instrument platform, the installation of power equipment platform with a variety of flexible and small load equipment, have no proper and low cost scheme for automatically leveling. In addition, some equipment need to adjust the levelness and horizontal position of platform at the same time, the current processing methods is to adjust the levelness automatically while adjust horizontal position manually. Due to being unable to realize full automation control, the adjustment is time-consuming and the control precision is not high. For the leveling of small equipments, considering the small load, the hydraulic leveling device is unfavorable, and using the adjustment of electromechanical has short leveling time, high precision leveling and reliability, etc. This work of this paper is to design a kind of small platform electromechanical automatic adjustment device using PLC as control core . The device is mainly applied to power equipment of smaller load or some small devices with the higher levelness requirements, which have some advantages, such as small sizes , flexible structure and high precision. 


\section{CONTROL PRINCIPLE}

The small device can automatically adjust the height, levelness and horizontal position. So the control principles are analyzed first to meet the need of the platform.

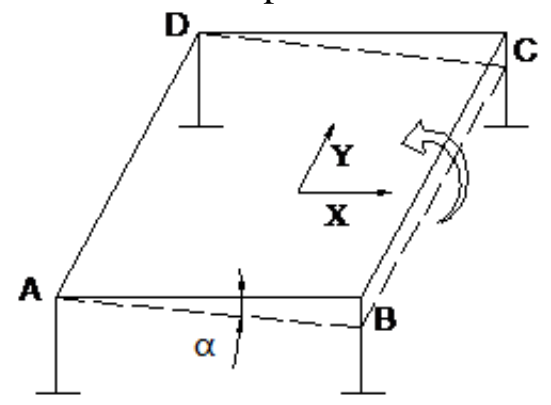

Figure1. Adjustment principle at x direction

As is shown in figure 1, the four leg support leveling system is adopted, with high bearing capacity, strong ability to resist capsizing and control is relatively easy, etc。In terms of this leveling system, some factors must be considered comprehensively, such as high leveling precision, high efficiency of leveling, strong stability of adjustment, etc. In terms of adjustment principle, it can adjust every leg individually also adjust the platform in direction respectively, etc. Due to the complexity of each leg adjustment and control separately, it can easily cause a virtual leg. So this paper adopts a leveling method of one-direction rise leg. Using the method, the highest point of platform is fixed ,then according to the $\mathrm{X}$ direction and $\mathrm{Y}$ direction in turn, only to adjust the lower side in all directions until levelness meets the need leveling[3][4]。 The specific leveling process is as follows: First to level the $\mathrm{X}$ direction, by reading the value of the angle of $\alpha$ horizontal Angle sensor measured, if the $\alpha<0$, it can be concluded the X positive axis are lower than the negative. Then the this information through the AD converter is sent to PLC controller to fix the two points $\mathrm{A}$ and $\mathrm{D}$, and elevate the other two points $\mathrm{B}$ and $\mathrm{C}$ at the same time, and made the BC rotate counterclockwise around $\mathrm{AD}$, so that decreasing the value of angle of $\alpha$ until meet the required levelness at $\mathrm{X}$ direction. Otherwise, fix the BC ,Let the AD rotate clockwise around $\mathrm{BC}$ to until the value of $\alpha$ arrive the required accuracy. Judgment at $\mathrm{Y}$ direction is similar to $\mathrm{X}$ direction ,and the adjustment method is similar too.

\section{DESIGN OF CONTROL STRUCTURE}

The hybrid closed loop structure is adopted to assure the leveling precision as is shown in figure 2 .

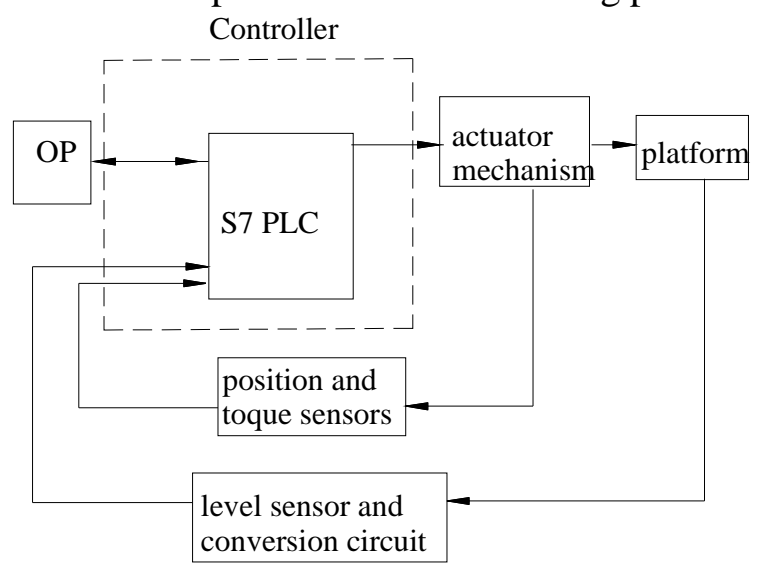

Figure 2. General frame of leveling system 
The small platform automatic leveling device is mainly composed of PLC control module, angle sensor, stepper motor, stepper motor drives, limit switch, virtual legs detection switch, LCD touch panel and screw mechanism. This equipment mainly includes two control functions, namely the height adjustment and horizontal position adjustment. In terms of adjustment of height and level precision, considering the required leveling speed is not high, in order to make the structure of equipment compact and smooth transmission, the ball screw vice is adopted as a lifting mechanism. To choose a small volume motor , combining motor with screw use elastic coupling, to minimize the power and torque of drive motor. Due to equipment requires adjustment of the horizontal position at the same time, considering the leveling equipment requires light weight, small volume and easy structure, meanwhile the position accuracy require is not high, the two sets ball screw and rolling guide is used as horizontal position adjustment mechanism. Because the general PLC only has two high frequency pulse output interface, but this system need control six stepper motors at the same time, therefore, a positioning control module is used to expand the PLC, which can control two stepper motor to realize two axis positioning, the $\mathrm{X}$ axis and $\mathrm{Y}$ axis. In addition, the system adopts the method of adjusting the levelness through control the four step motor for the supported leg. The method based on the horizontal inclination angle divide two step motor at $\mathrm{X}$ direction or $\mathrm{Y}$ direction at a period of same time, raise legs to adjust the horizontal of the direction and then adjust the other direction in the same method. It can be known that the two motors drive at the same time every time, same pulse, control of a high frequency pulse output interface is enough, only using the contact in the peripheral circuit design can be.

\section{SOFTWARE DESIGN}

There may be interference that will produce certain effect on horizontal precision of another direction as adjustment one direction to level when utilizing the method of elevating leg at one-direction, so using the method of loop control leveling when this approach is operated, which Can meet the required precision after a few loops, the process is shown in figure 3 [4]. As shown in the figure 3, first read the required value of the platform the levelness, adjust the required horizontal position automatically through the two-axis control and then adjust the levelness of the platform by adjusting the four legs 。 Due to the use of one-way rise leg adjustment method, even if there is interference and the coupling while the process of leveling, the highest point of the platform is changed, to achieve the purpose of leveling, through single direction adjustment in turn also 。 Strategies of rising one-way leg leveling follow the principle of leg go up only in the process of leveling strictly to ensure the accuracy and stability of the leveling and eliminate the coupling and interference in the process of leveling effectively at the same time. This method don't have to seek the highest point, also to avoid the disadvantage of specific control algorithm is difficult to achieve because each leg of displacement and speed are not identical when adjustment of more points, reducing the system running time and workload of programming 。 


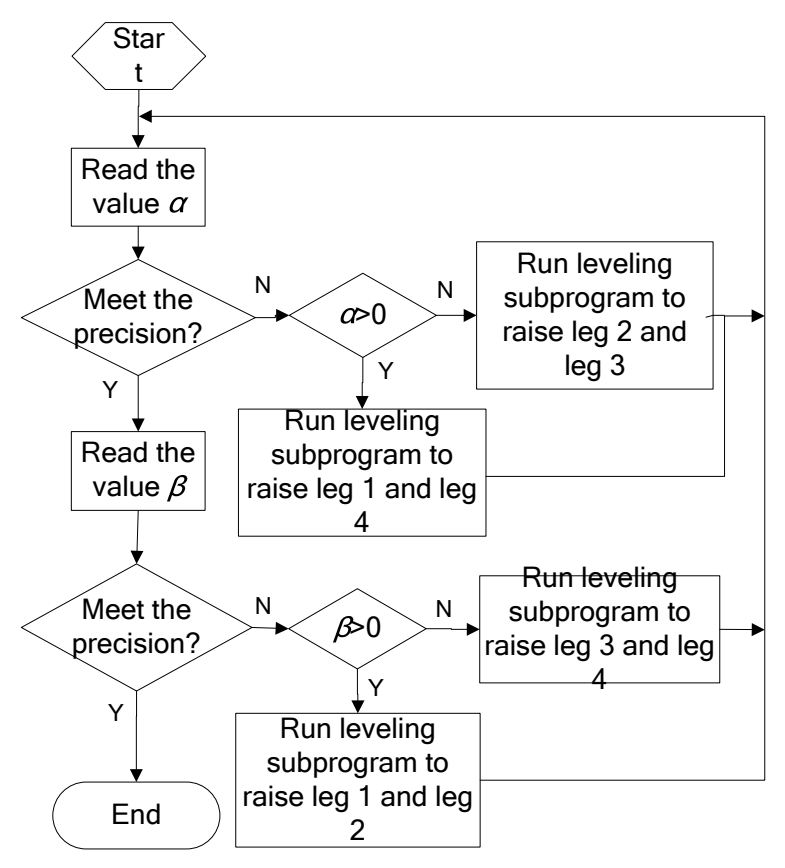

Figure 3. Flow chart of platform automatic adjustment

\section{EXPERIMENT AND CONCLUSION}

The main functions of the system are as follow: first, through the serial port to achieve the display of the main menu, to receives the touch screen button information and carries out the corresponding function processing; detection the state of the switch of four virtual legs and carrying out the corresponding treatment; detection the status of the upper and lower limit switch and carrying out the corresponding processing; sampling angle value of the two axis angle sensor and calibration.

The device has passed on-site test , the result shows that the system is stable, its leveling accuracy is less than 0.5 degrees, leveling time is less than $2 \mathrm{~min}$; the height, horizontal and horizontal position of the small platform are realized simultaneously, It can save the installation cost of small equipment effectively, time saving.

\section{ACKNOWLEDGMENT}

The work is funded by the scientific research fund of Wuhan Polytechnic University (2016y06).

\section{REFERENCES}

1. Ni Jiangsheng and Zhai Yujian, "Horizontal Adjustment of Radar Antenna Truck,” Measurement \& control technology, vol. 13, no. 4, Ju. 1994, pp. 36-39

2. Lin Xiao, Jiang Yong feng - "P87C591 SCM application in hydraulic leveling system for vehicle platform ," J. the modern electronic technology, 2006,18: 152 -156

3. Feng Yi and Chen Baiji, "Control system of automatically leveling of vehicle-borne radar," J. Hua zhong Univ of Sci. \& Tech. (Nature Science Edition), vol. 32, no. 6, Jun. 2004, pp. 65-68.

4. Yang Hongjun and Li Gangyan, "Study on leveling method and control technology of a vehicle-borne platform,” Machinery Design \& manufacture, no. 12, Dec. 2008, pp. 134-136. 